\title{
Investigations of the Riemann solver with internal reconstruction (RSIR) for the Euler equations
}

\author{
Alexandre Chiapolino ${ }^{1 \mathrm{a}}$, Richard Saurel ${ }^{2 \mathrm{a}, \mathrm{b}}$, Eleuterio Toro ${ }^{3 \mathrm{c}}$ \\ ${ }^{a}$ RS2N, Chemin de Gaumin, Saint-Zacharie 83640, France \\ ${ }^{b}$ Aix Marseille Univ, CNRS, Centrale Marseille, LMA, Marseille, France \\ ${ }^{c}$ Laboratory of Applied Mathematics DICAM, University of Trento, Italy
}

\begin{abstract}
The Riemann solver with internal reconstruction (RSIR) of Carmouze et al. (2019) is investigated, revisited and improved for the Euler equations. In this reference, the RSIR approach has been developed to address the numerical resolution of the non-equilibrium two-phase flow model of Saurel et al. (2017). The main idea is to reconstruct two intermediate states from the knowledge of a simple and robust intercell state such as HLL, regardless of the number of waves present in the Riemann problem. Such reconstruction improves significantly the accuracy of the HLL solution, preserves robustness and maintains stationary discontinuities. Consequently, when dealing with complex flow models, such as the aforementioned one, RSIR-type solvers are quite easy to derive compared to HLLC-type ones that may require a tedious analysis of the governing equations across the different waves. In the present contribution, the RSIR solver of Carmouze et al. (2019) is investigated, revisited and improved with the help of thermodynamic considerations, making a simple, accurate, robust and positive Riemann solver. It is also demonstrated that the RSIR solver is strictly equivalent to the HLLC solver of of Toro et al. (1994) for the Euler equations when the Rankine-Hugoniot relations are used. In that sense, the RSIR approach recovers the HLLC solver in some limit as well as the HLL one in another limit and can be simplified at different levels when complex systems of equations are addressed. For the sake of clarity and simplicity, the derivations are performed in the context of the one-dimensional Euler equations. Comparisons and validations against the conventional HLLC solver and exact solutions are presented.
\end{abstract}

Keywords: Riemann solver, RSIR, HLL, HLLC, hyperbolic

1 alexandre.chiapolino@rs2n.eu

2 richard.saurel@univ-amu.fr

${ }^{3}$ eleuterio.toro@unitn.it 


\section{Introduction}

Derivation of an appropriate Riemann solver for complex flow models is a difficult task. Many waves may be present, some eigenvalues may have large multiplicity and some of the equations may be non-conservative. Examples of such models are the MHD equations (Balsara 5 et al. (2012) [1]), compressible solid-fluid models (Gavrilyuk et al. (2008) [2]), non-equilibrium two-phase flow models such as the one of Saurel et al. (2017) [3], as well as the GodunovPeshkov-Romenski (GPR) model (Peshkov et al. (2019) [4]). Also, when dealing with material interfaces, large density jumps may be present and volume fraction as well as density positivity is mandatory. Same requirement is needed for high speed flows, where vacuum conditions may - appear. It seems that the most appropriate Riemann solver in these conditions is the HLLC solver of Toro et al. (1994) [5]. However, for specific models, its derivation may be non-trivial [6], [7].

In Carmouze et al. (2019) [8] an alternative is given and a new Riemann solver with internal reconstruction (RSIR) is designed. It relies on the following observation. It is usually quite easy to derive a single intermediate state solver, such as Rusanov (1961) [9] or HLL (Harten et al. (1983) [10] ) even for complicated flow models. These solvers are very robust and positive but too dissipative for transport and stationary contact waves. Extensions of the HLL solver to include more wave information have been developed in Einfeldt et al. (1991) [11], Toro et al. (1994) [5], Linde (2002) [12] and Dumbser and Balsara (2016) [13].

In Carmouze et al. (2019) [8] the single intermediate solution is used to rebuild two intermediate states, thanks to an additional relation. These two intermediate states contain in most situations enough information to improve significantly accuracy and preserve robustness.

The contribution of Carmouze et al. (2019) [8] was mainly motivated by the numerical approximation of the non-equilibrium two-phase flow model of Saurel et al. (2017) [3] that involves 25 a series of theoretical challenges as it is hyperbolic degenerate, presents non-conservative terms and exhibit non self-similar solutions. The complexity of the corresponding model prompted the authors to develop a solver based on internal reconstruction of intermediate states, computed from a simple and robust intercell state.

Thanks to the RSIR approach, stationary interfaces are maintained and numerical dissipation is reduced while circumventing the difficulties related to the construction of a HLLC-type solver. Chiapolino and Saurel (2020) [14] provide illustrative results of the RSIR solver in this two-phase flow context.

In Carmouze et al. (2019) [8], thermodynamic considerations have been introduced in the RSIR solver for the Euler equations and a similar treatment has been developed to address 5 numerical resolution of Saurel et al. (2017) [3] model.

The underlying philosophy of this approach relies on the assumption that most of the physics is present in the two extreme waves and only one contact wave, that has to be identified. If the contact wave cannot be defined clearly, the method becomes irrelevant. But it seems that in most flow models such as the Euler equations and the above-mentioned models, identification of the contact wave is possible and often easy.

The remaining waves, when present, are captured by the scheme during computations even if they are omitted in the Riemann problem. This is the same philosophy as the Rusanov and HLL 
solvers, except that an extra intermediate wave is added, preserving stationary discontinuities. The basic concept of the RSIR approach relies on three ingredients:

- A simple and robust intermediate solution state such as HLL or Rusanov;

- A consistency relation resulting from the integral form of conservation laws in a control volume containing a three-wave structure of a Riemann problem (two extreme waves and one contact wave);

- A heuristic relation linking the jumps of the two intermediate states across the contact wave.

The consistency equation is essential for the RSIR approach and is well-established for conservation laws. It links the simple and robust but single-state HLL (or Rusanov) solution and the two intermediate solution states. Its practical use requires nonetheless the heuristic relation that offers multiple choices depending on the properties of the governing equations.

In the present paper, the heuristic relation is addressed through thermodynamic considerations. This approach has been introduced in Carmouze et al. (2019) [8]. It is here investigated, revisited and improved. Second, it is demonstrated that the RSIR limit recovers the HLLC solver for the Euler equations when the heuristic relation is addressed through the Rankine-Hugoniot relations. Computed results confirm this observation. In that sense, the RSIR approach recovers the HLLC solver in some limit as well as the HLL one in another limit and can be simplified at different levels when complex systems of equations are addressed.

For the sake of clarity and simplicity, only the one-dimensional Euler equations are considered. Comparisons with the conventional HLLC solver and exact solutions are then available. The aim is to illustrate the simplicity, accuracy, robustness and flexibility of the RSIR solver.

This paper is organized as follows. The 1D Euler equations are briefly introduced in Section 2 along with the HLL and HLLC solvers as they correspond to the two limits of the RSIR solver. The RSIR solver is then addressed in Section [3. Its discrete entropy production is addressed in Section 4. The RSIR limit is then examined in Section 5 showing that the HLLC solver is recovered. Numerical results are provided in Section 6. They show that the RSIR solver provides results in close agreement with those of the HLLC solver while being relatively simple and flexible. Conclusions are finally given in Section 7.

\section{The HLL and HLLC Riemann solvers for the Euler equations}

The 1D Euler equations of compressible fluids consist in a system of conservation laws,

$$
\frac{\partial \mathbf{U}}{\partial t}+\frac{\partial \mathbf{F}}{\partial x}=0
$$

where $\mathbf{U}=(\rho, \rho u, \rho E)^{T}$ is the vector of conservative variables and $\mathbf{F}=\left(\rho u, \rho u^{2}+p,(\rho E+p) u\right)^{T}$ the corresponding flux vector. The notations are conventional in fluid dynamics, $\rho$ denotes the density, $u$ the velocity and $E=e+\frac{1}{2} u^{2}$ the total energy. The pressure $p$ is given by a convex equation of state (EOS), as a function for example of internal energy $e$ and density $\rho$. The idealgas EOS is used in the present contribution as $p(\rho, e)=(\gamma-1) \rho e$ where $\gamma=1.4$ represents the 
specific heat ratio. This EOS is retained for the sake of conciseness only. The numerical method addressed in the present work is valid for any convex EOS. This system is strictly hyperbolic with wave speeds $\lambda_{1}=u, \lambda_{2}=u-c$ and $\lambda_{3}=u+c$. The sound speed is defined as $c=\sqrt{\left(\frac{\partial p}{\partial \rho}\right)_{s}}$ where $s$ denotes the entropy. In the context of the ideal-gas EOS, the sound speed reads $c(p, \rho)=\sqrt{\frac{\gamma p}{\rho}}$.

The RSIR solver is based on the HLL (Harten et al. (1983) [10]) approximate solution, or its simplified version due to Rusanov (1961) [9]. It aims to construct a robust Riemann solver with limited dissipation, producing results similar to those provided by the HLLC solver.

The HLL and HLLC Riemann solvers are briefly recalled in the following. As will be seen further, the solutions provided by those two approximate Riemann solvers are precisely the two limits of the RSIR solver. It is consequently necessary to introduce the HLL and HLLC solvers beforehand. For a detailed presentation, the reader is referred to Toro's textbook (2009) [15], particularly Chapter 10 of this reference.

\section{HLL and HLLC Riemann solvers}

The approximate HLL solver requires estimates for two extreme waves emerging from the initial discontinuity. It results from the integration of the corresponding equations over a twowave Riemann problem.

A more accurate method is the HLLC solver, developed by Toro et al. (1994) [5]. This method assumes a three-wave model for the structure of the Riemann problem, resulting in better resolution of intermediate waves. The two wave structures (HLL and HLLC) of the Riemann problem are depicted in Fig. 1. 


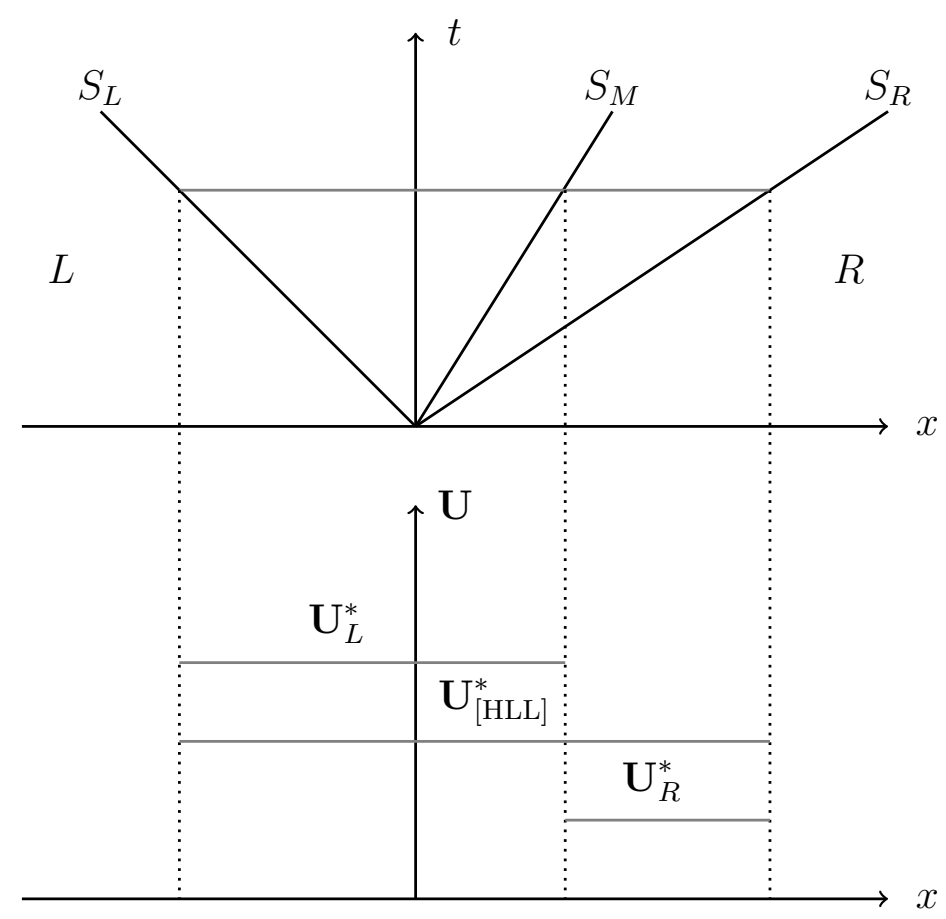

Figure 1: Schematic representation in the $(x, t)$ diagram of the HLL, HLLC and RSIR solvers. The HLL solver assumes a two-wave structure $\left(S_{L}\right.$ and $\left.S_{R}\right)$ while the intermediate wave $S_{M}$ is restored with the HLLC solver. The RSIR solver is based on the HLL single state solution complemented by a consistency relation reconstructing two intermediate states $\mathbf{U}_{L}^{*}, \mathbf{U}_{R}^{*}$ and a heuristic relation linking the jump of the two intermediate states across the contact wave $S_{M}$.

The central idea of the HLL solver is to assume a wave configuration that consists of two waves separating three constant states. The extreme waves denoted $S_{L}$ and $S_{R}$ are estimated following Davis (1988) [16],

$$
S_{L}=\min \left(u_{L}-c_{L}, u_{R}-c_{R}\right) \quad \text { and } \quad S_{R}=\max \left(u_{L}+c_{L}, u_{R}+c_{R}\right),
$$

where indexes $L$ and $R$ denote the left and right states at a given cell boundary. Note that a subsonic wave pattern is assumed in the present work, i.e. $S_{L}<0$ and $S_{R}>0$. These simple wave speed estimates yield accurate results. Moreover, they are convenient for complicated EOS and more sophisticated models than the Euler equations.

Both HLL and HLLC consider waves as discontinuities. Related jump conditions are the well-known Rankine-Hugoniot (RH) conditions:

$$
\mathbf{F}_{k}^{*}=\mathbf{F}_{k}+S_{k}\left(\mathbf{U}_{k}^{*}-\mathbf{U}_{k}\right), \quad k=L, R,
$$

where $S_{k}$ denotes the speed of the considered wave $k$. Note that the states involved in relation (2.3) are spatial integral averages. So strictly speaking these are not the classical RankineHugoniot conditions connecting limiting values left and right of a discontinuity but rather the "Averaged Rankine-Hugoniot" conditions. However the specification "Averaged" will be omitted in the rest of the paper. 
In the HLL solver, no distinction is made between states $\mathbf{U}_{R}^{*}$ and $\mathbf{U}_{L}^{*}$. The solution state in the HLL approximation reads,

$$
\mathbf{U}_{[\mathrm{HLL}]}^{*}=\frac{\mathbf{F}_{R}-\mathbf{F}_{L}+S_{L} \mathbf{U}_{L}-S_{R} \mathbf{U}_{R}}{S_{L}-S_{R}} .
$$

The resulting HLL Riemann solver forms the basis of very efficient and robust approximate Godunov-type methods. However, as the intermediate wave is omitted, the HLL solver produces more dissipation than the HLLC one. This is not problematic for fast flows as discontinuity are captured and in general maintained sharp enough during sufficiently long time, but becomes problematic for slower flows. Particularly, the HLL solver is unable to maintain discontinuities at rest.

The HLLC scheme is a modification of the HLL scheme, whereby the missing contact in the Euler equations is restored. The solutions for the two intermediate state vectors $\mathbf{U}_{L}^{*}$ and $\mathbf{U}_{R}^{*}$ are sought. Similarly to the HLL solver, where the Rankine-Hugoniot relations are used across the two extreme waves, the same jump conditions are used across the intermediate wave yielding contact discontinuity conditions:

$$
\left\{\begin{array}{l}
p_{L}^{*}=p_{R}^{*}=p^{*} \\
u_{L}^{*}=u_{R}^{*}=u^{*}=S_{M} .
\end{array}\right.
$$

As the extreme waves $S_{L}$ and $S_{R}$ are known from (2.2), algebraic manipulations of the mass and momentum Rankine-Hugoniot relations (2.3) provide the pressure solutions in the left and right perturbed states,

$$
p_{k}^{*}=p_{k}+\rho_{k}\left(S_{k}-u_{k}\right)\left(S_{M}-u_{k}\right), \quad k=L, R .
$$

The equality of the pressures allows determination of the intermediate speed $S_{M}$ as a function of speeds $S_{L}$ and $S_{R}$, namely,

$$
S_{M}=u_{L}^{*}=u_{R}^{*}=\frac{p_{R}-p_{L}+(\rho u)_{L}\left(S_{L}-u_{L}\right)-(\rho u)_{R}\left(S_{R}-u_{R}\right)}{\rho_{L}\left(S_{L}-u_{L}\right)-\rho_{R}\left(S_{R}-u_{R}\right)}=\frac{U_{[\mathrm{HLL}]}^{*, \text { momentum }}}{U_{[\mathrm{HLL}]}^{*, \text { mass }}} .
$$

The two intermediate solution states are computed with the help of the Rankine-Hugoniot relations $(2.3)$ and the corresponding values $p_{L}^{*}$ and $p_{R}^{*}$. The solutions can be written concisely as,

$$
\mathbf{U}_{k[\mathrm{HLLC}]}^{*}=\frac{S_{k} \mathbf{U}_{k}-\mathbf{F}_{k}+p_{k}^{*} \mathbf{D}}{S_{k}-S_{M}} \quad \text { with } \quad \mathbf{D}=\left[0,1, S_{M}\right]^{T}, \quad k=L, R .
$$

The numerical fluxes, solution of the Riemann problem, are provided by Eq. (2.3) according to the Rankine-Hugoniot relations across the left $S_{L}$ or right $S_{R}$ wave depending on the sign of the contact wave $S_{M}$ speed. 
Unlike the HLL solver, the HLLC solver is able to maintain stationary discontinuities. However, the construction of a HLLC-type solver may be quite difficult for more sophisticated equation systems such as two-phase flow models or magnetohydrodynamics equations where many waves are present in the Riemann problem.

Recently, the authors developed in Carmouze et al. (2019) [8] a Riemann Solver with Internal Reconstruction (RSIR). It is based on the HLL single state solution, a consistency relation reconstructing two intermediate states $\mathbf{U}_{L}^{*}, \mathbf{U}_{R}^{*}$ and a heuristic relation linking the jump of the two intermediate states across the contact wave $S_{M}$. In the present paper, the heuristic relation is revisited and improved.

\section{Riemann Solver with Internal Reconstruction (RSIR)}

The RSIR solver is based on internal reconstruction of intermediate states, computed from a simple and robust intercell state, such as Rusanov (1961) [9] or HLL (Harten et al. (1983) [10]). The HLL solution state $\mathbf{U}_{[\mathrm{HLL}]}^{*}$ is known from Eq. (2.4) and the contact wave speed $S_{M}$ is also known from the HLL solution (Eq. (2.7)). The extreme waves $S_{L}$ and $S_{R}$ are known with the help of Davis' estimates (2.2).

The aim is now to construct two intermediate states $\mathbf{U}_{L}^{*}$ and $\mathbf{U}_{R}^{*}$ as illustrated in Fig. 1. The average HLL state and the two intermediate solution states are linked through the consistency relation (illustrated in Fig. 1),

$$
\left(S_{R}-S_{L}\right) \mathbf{U}_{[\mathrm{HLL}]}^{*}=\left(S_{R}-S_{M}\right) \mathbf{U}_{R}^{*}+\left(S_{M}-S_{L}\right) \mathbf{U}_{L}^{*}
$$

This consistency relation can be rewritten as,

$$
\mathbf{U}_{[\mathrm{HLL}]}^{*}=\omega_{R} \mathbf{U}_{R}^{*}+\omega_{L} \mathbf{U}_{L}^{*}
$$

with

$$
\omega_{R}=\frac{S_{R}-S_{M}}{S_{R}-S_{L}} \quad \text { and } \quad \omega_{L}=\frac{S_{M}-S_{L}}{S_{R}-S_{L}} .
$$

Relation (3.2) involves two unknown states, $\mathbf{U}_{L}^{*}$ and $\mathbf{U}_{R}^{*}$. Consequently an extra relation, linking the jumps of the two intermediate states across the contact wave, is needed.

First let us introduce the heuristic of Linde (2002) [12],

$$
\mathbf{U}_{R}^{*}-\mathbf{U}_{L}^{*}=\beta\left(\mathbf{U}_{R}-\mathbf{U}_{L}\right) .
$$

In this relation, $\beta$ represents a viscosity parameter, $0 \leq \beta \leq 1$. When $\beta$ is taken equal to zero, the HLL approximation is recovered. When $\beta=1$ the reconstruction tends to the HLLC representation but is not equivalent, as interface conditions (2.5) are ignored in the Linde approach.

Relation (3.4) is then combined with Relation (3.2) resulting in,

$$
\left\{\begin{array}{l}
\mathbf{U}_{L[\text { Linde }]}^{*}=\mathbf{U}_{[\mathrm{HLL}]}^{*}-\omega_{R} \beta\left(\mathbf{U}_{R}-\mathbf{U}_{L}\right), \\
\mathbf{U}_{R[\text { Linde }]}^{*}=\mathbf{U}_{[\mathrm{HLL}]}^{*}+\omega_{L} \beta\left(\mathbf{U}_{R}-\mathbf{U}_{L}\right) .
\end{array}\right.
$$


With the help of Relation (3.5), corresponding fluxes are computed thanks to the RH relations

(2.3). As shown in Carmouze et al. (2019) [8], Eq. (3.5) sometimes yield correct results but large spurious oscillations may appear as well depending on the initial conditions.

This observation motivated reconsideration of the heuristic relation, now based on thermodynamic considerations, resulting in significant improvements and yielding robust and accurate solutions. The heuristic relation now reads,

$$
\mathbf{U}_{R}^{*}-\mathbf{U}_{L}^{*}=\Psi
$$

where $\Psi$ is the jump vector linking the two intermediate state $\mathbf{U}_{L}^{*}$ and $\mathbf{U}_{R}^{*}$. As previously, Relation (3.6) is combined with Eq. (3.2) resulting in,

$$
\left\{\begin{aligned}
\mathbf{U}_{L[\mathrm{RSIR}]}^{*} & =\mathbf{U}_{[\mathrm{HLL}]}^{*}-\omega_{R} \boldsymbol{\Psi}, \\
\mathbf{U}_{R[\mathrm{RSIR}]}^{*} & =\mathbf{U}_{[\mathrm{HLL}]}^{*}+\omega_{L} \boldsymbol{\Psi} .
\end{aligned}\right.
$$

Relation (3.7) is the cornerstone of the RSIR approach. It results from the combination of the consistency relation and the jump vector linking the two intermediate states. Equation (3.7) provides the solution of the two intermediate states, built upon the HLL solution. However, its practical use requires knowledge of the jump vector $\boldsymbol{\Psi}$. This task offers multiple choices depending on the properties of the governing equations.

It is important to note that only an approximate jump vector $\boldsymbol{\Psi}$ is needed to improve the HLL solution through Eq. (3.7). As will be seen further, the different components of the jump vector may sometimes be expressed directly, for example $\left(\rho_{R}^{*}-\rho_{L}^{*}\right)$, or two approximate variables may be obtained separately, for example $\rho_{R}^{*}$ and $\rho_{L}^{*}$, giving the jump by subtracting the two quantities. However, in the latest case, approximate variables such as $\rho_{R}^{*}$ and $\rho_{L}^{*}$ are not to be used as solutions but only as an approximation of the jump $\boldsymbol{\Psi}$, to be combined with the consistency relation.

Regardless the way the jump vector $\boldsymbol{\Psi}$ is computed, the two solution states $\mathbf{U}_{L}^{*}$ and $\mathbf{U}_{R}^{*}$ are determined through Eq. (3.7). The solutions are then built upon the HLL one and the approximation of the jump vector.

In the following, crude knowledge of the governing equations' properties is assumed in the aim to address complex flow models. The aim is then to approximate the jump vector $\boldsymbol{\Psi}$ from relations as simple and general as possible while involving relevant physics. As will be seen later in Section 5, when more details of the governing equations are available, the RSIR solver recovers the HLLC one through the introduction of the Rankine-Hugoniot relations in the jump vector $\Psi$

The approximation of the jump vector $\boldsymbol{\Psi}$ derived hereafter is based on two ingredients:

- Quasi-isentropic or barotropic variations across right- and left-facing waves;

- Insertion of interface conditions across the contact wave.

Although not strictly correct, in particular across strong shocks, thermodynamic evolutions through right-and left-facing waves are approximated as quasi-isentropic. 


\section{Mass relation}

Thermodynamic evolutions across the right- and left-facing waves are approximated through

200

sound speed definition $c^{2}=\left(\frac{\partial p}{\partial \rho}\right)_{s}$ as,

$$
\bar{c}^{2}=\frac{p_{L}^{*}-p_{L}}{\rho_{L}^{*}-\rho_{L}}=\frac{p_{R}^{*}-p_{R}}{\rho_{R}^{*}-\rho_{R}} .
$$

Such approximation has been used for example in Appendix A of [17] to approximate isentropes in tank boundary conditions, when dealing with sophisticated equations of state. The average square sound speed $\bar{c}^{2}$ is estimated as,

$$
\bar{c}^{2}=\max \left[c_{L}^{2}, c_{R}^{2}\right]
$$

As will be seen in Section 4, this estimate guarantees discrete entropy production. Note that

this is different from the original RSIR solver introduced in Carmouze et al. (2019) [8] where the average sound speed is computed with the help of a rough trapezoidal approximation, $\bar{c}=$ $\frac{1}{2}\left(c_{L}+c_{R}\right)$.

Across the contact wave $S_{M}$, the interface pressure condition reads,

$$
p_{L}^{*}=p_{R}^{*}=p^{*}
$$

Thus Eq. (3.8) becomes,

$$
\left\{\begin{array}{l}
p^{*}=p_{R}+\bar{c}^{2}\left(\rho_{R}^{*}-\rho_{R}\right), \\
p^{*}=p_{L}+\bar{c}^{2}\left(\rho_{L}^{*}-\rho_{L}\right),
\end{array}\right.
$$

Taking the difference of these two relations, the following one is obtained:

$$
\rho_{R}^{*}-\rho_{L}^{*}=\rho_{R}-\rho_{L}-\frac{p_{R}-p_{L}}{\bar{c}^{2}} .
$$

Relation (3.12) corresponds to a modification of the first component of the Linde's approximation (3.4). To maintain flexibility of the reconstruction method, parameter $\beta$ is reintroduced as,

$$
\rho_{R}^{*}-\rho_{L}^{*}=\beta\left(\rho_{R}-\rho_{L}+\frac{p_{L}-p_{R}}{\bar{c}^{2}}\right)=\Psi^{\text {mass }} .
$$

Parameter $\beta$ seems convenient to control numerical viscosity, for example to remove the contact wave when dealing with computations in extreme conditions. In all examples considered in the present paper, $\beta=1$, corresponding to the least dissipative version.

Equation (3.13) is an approximation of the mass jump between the two intermediate states. The solution densities are now determined with the help of the mass jump and the consistency 
relation (3.7),

$$
\left\{\begin{array}{l}
\rho_{L}^{*}=\rho_{[\mathrm{HLL}]}^{*}-\omega_{R} \beta\left(\rho_{R}-\rho_{L}+\frac{p_{L}-p_{R}}{\bar{c}^{2}}\right) \\
\rho_{R}^{*}=\rho_{[\mathrm{HLL}]}^{*}+\omega_{L} \underbrace{\beta\left(\rho_{R}-\rho_{L}+\frac{p_{L}-p_{R}}{\bar{c}^{2}}\right)}_{\Psi^{\text {mass }}} .
\end{array}\right.
$$

\section{Momentum relation}

Similar relations are deduced to approximate the momentum jump across the contact wave. As $u_{L}^{*}=u_{R}^{*}=S_{M}$, Eq. (3.13) implies the following relation,

$$
(\rho u)_{R}^{*}-(\rho u)_{L}^{*}=\beta\left(\rho_{R}-\rho_{L}+\frac{p_{L}-p_{R}}{\bar{c}^{2}}\right) S_{M}=\Psi^{\text {momentum }} .
$$

The solution momenta consequently read,

$$
\left\{\begin{array}{c}
(\rho u)_{L}^{*}=(\rho u)_{[\mathrm{HLL}]}^{*}-\omega_{R} \beta\left(\rho_{R}-\rho_{L}+\frac{p_{L}-p_{R}}{\bar{c}^{2}}\right) S_{M}, \\
(\rho u)_{R}^{*}=(\rho u)_{[\mathrm{HLL}]}^{*}+\omega_{L} \underbrace{\beta\left(\rho_{R}-\rho_{L}+\frac{p_{L}-p_{R}}{\bar{c}^{2}}\right) S_{M}}_{\Psi^{\text {momentum }}} .
\end{array}\right.
$$

\section{Energy relation}

As quasi-isentropic variations across right- and left-facing waves are supposed, the approximation of the energy jump is determined with the help of the first law of thermodynamics, expressed under the form of Gibbs' relation,

$$
d e=T \underbrace{d s}_{=0}-p d v
$$

where $T$ denotes the temperature, $s$ the entropy and $v=1 / \rho$ the specific volume. Thanks to the quasi-isentropic assumption, Gibbs' relation is approximated as,

$$
\left\{\begin{array}{l}
e_{L}^{*}=e_{L}-\bar{p}_{L}\left(v_{L}^{*}-v_{L}\right), \\
e_{R}^{*}=e_{R}-\bar{p}_{R}\left(v_{R}^{*}-v_{R}\right),
\end{array}\right.
$$

where $v_{L}^{*}=1 / \rho_{L}^{*}$ and $v_{R}^{*}=1 / \rho_{R}^{*}$ are known from Eq. (3.14). In Relation (3.18), $\bar{p}_{k}$ is an estimate of the average pressure across the corresponding wave. Multiple choices are available at this point. The most obvious ones are $\bar{p}_{k}=p_{k}$ with $k=L, R$ or $\bar{p}_{k}=p^{*}$ or a combination of them $\bar{p}_{k}=\frac{p_{k}+p^{*}}{2}$. As will be seen further, the analysis of the entropy production reports that only $\bar{p}_{k}=p^{*}$ is an admissible estimate. This estimate is determined from the average square sound speed as,

$$
p^{*}=p_{L}+\bar{c}^{2}\left(\rho_{L}^{*}-\rho_{L}\right)=p_{R}+\bar{c}^{2}\left(\rho_{R}^{*}-\rho_{R}\right) .
$$


This is another difference with the original RSIR solver introduced in Carmouze et al. (2019) [8] where the $\operatorname{EOS} e_{k}^{*}\left(p_{k}^{*}, \rho_{k}^{*}\right)$ is used to compute directly the energy jump.

In the present paper, the quasi-isentropic assumption is approximated through both sound speed definition and Gibbs' relation. The total energy jump is finally expressed thanks to Eqs. (3.14) and (3.18),

$$
(\rho E)_{R}^{*}-(\rho E)_{L}^{*}=\beta\left[\rho_{R}^{*}\left(e_{R}^{*}+\frac{1}{2} S_{M}^{2}\right)-\rho_{L}^{*}\left(e_{L}^{*}+\frac{1}{2} S_{M}^{2}\right)\right]=\Psi^{\text {energy }},
$$

where the $\beta$ parameter is reintroduced for the sake of generality. The solutions for total energies are now built upon the HLL ones as,

$$
\left\{\begin{array}{l}
(\rho E)_{L}^{*}=(\rho E)_{[\mathrm{HLL}]}^{*}-\omega_{R} \beta\left[\rho_{R}^{*}\left(e_{R}^{*}+\frac{1}{2} S_{M}^{2}\right)-\rho_{L}^{*}\left(e_{L}^{*}+\frac{1}{2} S_{M}^{2}\right)\right] \\
(\rho E)_{R}^{*}=(\rho E)_{[\mathrm{HLL}]}^{*}+\omega_{L} \beta \underbrace{}_{\Psi_{\text {energy }}\left[\rho_{R}^{*}\left(e_{R}^{*}+\frac{1}{2} S_{M}^{2}\right)-\rho_{L}^{*}\left(e_{L}^{*}+\frac{1}{2} S_{M}^{2}\right)\right]} .
\end{array}\right.
$$

The intermediate solution states are then fully determined for the Euler equations. They are computed upon the HLL solution with the help of the consistency relation and the jump vector,

$$
\left\{\begin{aligned}
\mathbf{U}_{L[\mathrm{RSIR}]}^{*} & =\mathbf{U}_{[\mathrm{HLL}]}^{*}-\omega_{R} \boldsymbol{\Psi} \\
\mathbf{U}_{R[\mathrm{RSIR}]}^{*} & =\mathbf{U}_{[\mathrm{HLL}]}^{*}+\omega_{L} \boldsymbol{\Psi}
\end{aligned}\right.
$$

\section{Solution fluxes}

Once states $\mathbf{U}_{L}^{*}$ and $\mathbf{U}_{R}^{*}$ are determined through (3.22) the various fluxes are computed through the Rankine-Hugoniot relations, according to the sign of $S_{M}$,

$$
\left\{\begin{array}{l}
\mathbf{F}_{R}^{*}=\mathbf{F}_{R}+S_{R}\left(\mathbf{U}_{R}^{*}-\mathbf{U}_{R}\right) \\
\mathbf{F}_{L}^{*}=\mathbf{F}_{L}+S_{L}\left(\mathbf{U}_{L}^{*}-\mathbf{U}_{L}\right)
\end{array}\right.
$$

The Riemann solver thus consists in Eq. (2.4) to compute the simple and robust HLL state, Eqs. (3.13), (3.15), (3.20) to approximate the jumps across the intermediate wave $S_{M}$ and Eq. (3.22) to construct the two intermediate states. Note that the solver does not require explicit formulation of the EOS.

\section{Entropy production}

The discrete entropy production of the RSIR solver is now addressed. During the construction of the jump vector $\Psi$ and particularly the energy component $\Psi^{\text {energy }}$, the following thermodynamic path is used,

$$
d e=-\bar{p} d v
$$


where $\bar{p}$ is an estimate of the average pressure.

The choice of $\bar{p}$ is dictated by the second law of thermodynamics. Let us insert the present thermodynamic path into Gibbs' relation,

$$
T d s=d e+p d v=-\bar{p} d v+p d v .
$$

The integration of the previous relation over the unperturbed state $(k=L, R)$ and the solution state $\left(\begin{array}{l}* \\ k\end{array}\right)$ reads,

$$
\int_{k}^{*_{k}} T d s=\int_{k}^{*_{k}}(p-\bar{p}) d v
$$

The right-hand side of Relation (4.3) represents the discrete entropy production across the extreme waves $S_{L}$ and $S_{R}$. With the RSIR solver, the pressure evolves across the extreme waves as,

$$
p(\rho)=p_{k}+\bar{c}^{2}\left(\rho-\rho_{k}\right) .
$$

Equation (4.3) consequently transforms to,

$$
\int_{k}^{*_{k}} T d s=\int_{k}^{*_{k}}\left(p_{k}+\bar{c}^{2}\left(\rho-\rho_{k}\right)-\bar{p}\right) d v .
$$

The discrete entropy production is satisfied if the right hand-side of Eq. (4.5) is non-negative.

Let us now introduce the pressure estimate as $\bar{p}=p^{*}$. Relation (4.5) becomes,

$\int_{k}^{*_{k}} T d s=\int_{k}^{*_{k}}\left(p_{k}-p^{*}\right) d v+\bar{c}^{2} \int_{k}^{*_{k}}\left(\frac{1}{v}-\frac{1}{v_{k}}\right) d v=\left(p_{k}-p^{*}\right)\left(v_{k}^{*}-v_{k}\right)+\bar{c}^{2}\left(\ln \left(\frac{v_{k}^{*}}{v_{k}}\right)-\frac{v_{k}^{*}}{v_{k}}+1\right)$.

However, the RSIR star pressure reads $p^{*}=p_{k}+\bar{c}^{2}\left(\frac{1}{v_{k}^{*}}-\frac{1}{v_{k}}\right)$. The preceding relation consequently transforms to,

$$
\int_{k}^{*_{k}} T d s=\bar{c}^{2}\left(\frac{\left(v_{k}^{*}-v_{k}\right)^{2}}{v_{k}^{*} v_{k}}+\ln \left(\frac{v_{k}^{*}}{v_{k}}\right)-\frac{v_{k}^{*}}{v_{k}}+1\right) .
$$

For the sake of simplicity, let us introduce $X=\frac{v_{k}^{*}}{v_{k}}$. The discrete entropy production is then satisfied if,

$$
f(X)=\frac{(X-1)^{2}}{X}+\ln (X)-X+1 \geq 0 .
$$

A simple mathematical function analysis shows that $f(X) \geq 0 \forall X \in] 0,+\infty$ [ with a minimum corresponding to $f(X=1)=0$.

The estimate $\bar{p}=p^{*}$ consequently satisfies the discrete entropy production making the RSIR solver entropy preserving. It is interesting to emphasize that the amount of discrete entropy is 
quantified by the averaged sound speed $\bar{c}^{2}$. For the sake of robustness, it is preferable to use the

largest value of $\bar{c}^{2}$. For this reason, the average sound speed is estimated as,

$$
\bar{c}^{2}=\max \left[c_{L}^{2}, c_{R}^{2}\right]
$$

as seen in Section 3 ,

It is also important to emphasize that the previous calculations lead to $f(X) \leq 0 \forall X \in$ ] $0,+\infty\left[\right.$ if $\bar{p}=p_{k}$ and $f(X) \geq 0$ for $\left.\left.X \in\right] 0,1\right]$ if $\bar{p}=\frac{p_{k}+p^{*}}{2}$. Those estimates are consequently rejected.

Note that the latest result is not surprising as it indicates that the discrete entropy production is satisfied only for $X=\frac{v_{k}^{*}}{v_{k}}=\frac{\rho_{k}}{\rho_{k}^{*}}<1$ corresponding to a compression. Indeed the introduction of $\bar{p}=\frac{p_{k}+p^{*}}{2}$ into Gibbs' relation $d e=-\bar{p} d v$ leads to $e_{k}^{*}-e_{k}+\frac{p_{k}+p^{*}}{2}\left(v_{k}^{*}-v_{k}\right)=0$ which is precisely the Hugoniot adiabat, valid for compressions only.

It consequently appears that $\bar{p}=p^{*}$ (computed with the help of Eq. (3.19)) is the only admissible estimate among the ones examined. Numerical experiments with the present RSIR solver are provided in Section [6 showing efficiency and robustness. However, before analyzing the corresponding results, let us address the limit of the RSIR solver. When more details of the equations are used, meaning they are available, the jump vector $\boldsymbol{\Psi}$ can be approximated with the help of the Rankine-Hugoniot relations. This task is addressed hereafter showing that the

\section{Limit of the RSIR solver}

The RSIR solution relies on Eq. (3.7), recalled hereafter,

$$
\left\{\begin{aligned}
\mathbf{U}_{L[\mathrm{RSIR}]}^{*} & =\mathbf{U}_{[\mathrm{HLL}]}^{*}-\omega_{R} \boldsymbol{\Psi} \\
\mathbf{U}_{R[\mathrm{RSIR}]}^{*} & =\mathbf{U}_{[\mathrm{HLL}]}^{*}+\omega_{L} \boldsymbol{\Psi} .
\end{aligned}\right.
$$

Unlike Section 3 where quasi-isentropic evolutions are assumed, the construction of the jump vector $\Psi$ is now addressed through the set of Rankine-Hugoniot relations.

Mass relation

The Rankine-Hugoniot mass relations read,

$$
\left\{\begin{array}{l}
\rho_{L}^{*}\left(S_{M}-S_{L}\right)=\rho_{L}\left(u_{L}-S_{L}\right) \\
\rho_{R}^{*}\left(S_{M}-S_{R}\right)=\rho_{R}\left(u_{R}-S_{R}\right) .
\end{array}\right.
$$

The following jump relation consequently arises,

$$
\rho_{R}^{*}-\rho_{L}^{*}=\rho_{R} \frac{u_{R}-S_{R}}{S_{M}-S_{R}}-\rho_{L} \frac{u_{L}-S_{L}}{S_{M}-S_{L}} .
$$

Parameter $\beta$ is introduced as before for the sake of generality,

$$
\rho_{R}^{*}-\rho_{L}^{*}=\beta\left(\rho_{R} \frac{u_{R}-S_{R}}{S_{M}-S_{R}}-\rho_{L} \frac{u_{L}-S_{L}}{S_{M}-S_{L}}\right)=\Psi^{\mathrm{mass}} .
$$




\section{Momentum relation}

Thanks to the previous mass jump and the interface condition across the intermediate wave $S_{M}$, the momentum jump directly reads,

$$
(\rho u)_{R}^{*}-(\rho u)_{L}^{*}=\beta\left(\rho_{R} \frac{u_{R}-S_{R}}{S_{M}-S_{R}}-\rho_{L} \frac{u_{L}-S_{L}}{S_{M}-S_{L}}\right) S_{M}=\Psi^{\text {momentum }} .
$$

\section{Energy relation}

Combination of the mass, momentum and energy Rankine-Hugoniot relations provide after some algebraic manipulations,

$$
\left\{\begin{array}{c}
(\rho E)_{L}^{*}=\frac{(\rho E)_{L}\left(u_{L}-S_{L}\right)+p_{L} u_{L}-p_{L}^{*} S_{M}}{S_{M}-S_{L}}, \\
(\rho E)_{R}^{*}=\frac{(\rho E)_{R}\left(u_{R}-S_{R}\right)+p_{R} u_{R}-p_{R}^{*} S_{M}}{S_{M}-S_{R}},
\end{array}\right.
$$

where the star pressure is provided by the HLL solution, Eq. (2.6). With the help of these relations, a jump relation across the contact wave is obtained,

$(\rho E)_{R}^{*}-(\rho E)_{L}^{*}=\beta\left(\frac{(\rho E)_{R}\left(u_{R}-S_{R}\right)+p_{R} u_{R}-p_{R}^{*} S_{M}}{S_{M}-S_{R}}-\frac{(\rho E)_{L}\left(u_{L}-S_{L}\right)+p_{L} u_{L}-p_{L}^{*} S_{M}}{S_{M}-S_{L}}\right)=\Psi^{\mathrm{energy}}$.

The jump vector $\boldsymbol{\Psi}$ across the contact wave is now approximated with the help of RankineHugoniot relations. The solution vectors (5.1) are then to be compared to the HLLC solutions. Let us first recall the solution of HLLC solver for the Euler equations (see [15] for details),

$$
\mathbf{U}_{k[\mathrm{HLLC}]}^{*}=\frac{S_{k} \mathbf{U}_{k}-\mathbf{F}_{k}+p_{k}^{*} \mathbf{D}}{S_{k}-S_{M}} \quad \text { with } \quad \mathbf{D}=\left[0,1, S_{M}\right]^{T}, \quad k=L, R
$$

with

$$
p_{k}^{*}=p_{k}+\rho_{k}\left(S_{k}-u_{k}\right)\left(S_{M}-u_{k}\right),
$$

and

$$
S_{M}=u_{L}^{*}=u_{R}^{*}=\frac{p_{R}-p_{L}+(\rho u)_{L}\left(S_{L}-u_{L}\right)-(\rho u)_{R}\left(S_{R}-u_{R}\right)}{\rho_{L}\left(S_{L}-u_{L}\right)-\rho_{R}\left(S_{R}-u_{R}\right)}=\frac{U_{[\mathrm{HLL}]}^{* \text {,momentum }}}{U_{[\mathrm{HLL}]}^{*, \text { mass }}} .
$$

With the RSIR solver, solution states are given by,

$$
\left\{\begin{aligned}
\mathbf{U}_{L[\mathrm{RSIR}]}^{*} & =\mathbf{U}_{[\mathrm{HLL}]}^{*}-\omega_{R} \Psi \\
\mathbf{U}_{R[\mathrm{RSIR}]}^{*} & =\mathbf{U}_{[\mathrm{HLL}]}^{*}+\omega_{L} \Psi
\end{aligned}\right.
$$

Examination of the RSIR solution state vector $\mathbf{U}_{L[\mathrm{RSIR}]}^{*}$ is now addressed, 


\section{Mass solution}

The mass solution reads,

$$
\rho_{L[\mathrm{RSIR}]}^{*}=\underbrace{\left(\frac{\rho_{R} u_{R}-\rho_{L} u_{L}+S_{L} \rho_{L}-S_{R} \rho_{R}}{S_{L}-S_{R}}\right)}_{\rho_{[\mathrm{HLL}]}^{*}}-\underbrace{\left(\frac{S_{R}-S_{M}}{S_{R}-S_{L}}\right)}_{\omega_{R}} \underbrace{\beta\left(\rho_{R} \frac{u_{R}-S_{R}}{S_{M}-S_{R}}-\rho_{L} \frac{u_{L}-S_{L}}{S_{M}-S_{L}}\right)}_{\Psi^{\text {mass }}} .
$$

Equation (5.12) may be written as,

$$
\rho_{L[\mathrm{RSIR}]}^{*}=\frac{\rho_{R}\left(u_{R}-S_{R}\right)}{S_{L}-S_{R}}-\frac{\rho_{L}\left(u_{L}-S_{L}\right)}{S_{L}-S_{R}}-\beta \frac{\rho_{R}\left(u_{R}-S_{R}\right)}{S_{L}-S_{R}}+\beta\left(\frac{S_{R}-S_{M}}{S_{R}-S_{L}}\right) \rho_{L} \frac{u_{L}-S_{L}}{S_{M}-S_{L}} .
$$

When $\beta=0$, the HLL solution is recovered. When $\beta=1$, Eq. (5.13) reduces to,

$$
\rho_{L[\mathrm{RSIR}]}^{*}=\frac{\rho_{L}\left(u_{L}-S_{L}\right)}{S_{M}-S_{L}},
$$

which is precisely $\rho_{L[\mathrm{HLLC}]}^{*}$ according to Eq. (5.8). The density solution is consequently merged with the HLLC solver one.

\section{Momentum solution}

Combination of Eqs. (5.8) and (5.9) gives for the momentum,

$$
(\rho u)_{L[\mathrm{HLLC}]}^{*}=\frac{\rho_{L}\left(u_{L}-S_{L}\right)}{S_{M}-S_{L}} S_{M}=\rho_{L[\mathrm{HLLC}]}^{*} S_{M} .
$$

RSIR formula is now expanded for comparison with the HLLC solution,

$$
\begin{aligned}
(\rho u)_{L[\mathrm{RSIR}]}^{*}= & \underbrace{\frac{(\rho u)_{R}\left(u_{R}-S_{R}\right)-(\rho u)_{L}\left(u_{L}-S_{L}\right)+p_{R}-p_{L}}{S_{L}-S_{R}}}_{(\rho u)_{[\mathrm{HLL}]}^{*}} \\
& -\underbrace{\left(\frac{S_{R}-S_{M}}{S_{R}-S_{L}}\right)}_{\omega_{R}} \underbrace{\beta\left(\rho_{R} \frac{u_{R}-S_{R}}{S_{M}-S_{R}}-\rho_{L} \frac{u_{L}-S_{L}}{S_{M}-S_{L}}\right) S_{M}}_{\Psi^{\text {momentum }}} .
\end{aligned}
$$

After some simplifications it becomes,

$$
(\rho u)_{L[\mathrm{RSIR}]}^{*}=\left(\frac{\rho_{R}\left(u_{R}-S_{R}\right)}{S_{L}-S_{R}}\right)\left(u_{R}+\beta S_{M}\right)-\left(\frac{\rho_{L}\left(u_{L}-S_{L}\right)}{S_{L}-S_{R}}\right)\left(u_{L}+\beta S_{M} \frac{S_{R}-S_{M}}{S_{M}-S_{L}}\right)+\frac{p_{R}-p_{L}}{S_{L}-S_{R}} .
$$

The last term expresses as,

$$
\frac{p_{R}-p_{L}}{S_{L}-S_{R}}=\frac{\rho_{L}\left(S_{L}-u_{L}\right)\left(S_{M}-u_{L}\right)+\rho_{R}\left(S_{R}-u_{R}\right)\left(u_{R}-S_{M}\right)}{S_{L}-S_{R}},
$$


determined from the contact wave speed estimate formula (5.10). The RSIR star left momentum jump relation now becomes,

$$
(\rho u)_{L[\mathrm{RSIR}]}^{*}=\frac{\rho_{R}\left(u_{R}-S_{R}\right)}{S_{L}-S_{R}}\left[u_{R}-\beta S_{M}-\left(u_{R}-S_{M}\right)\right]-\frac{\rho_{L}\left(u_{L}-S_{L}\right)}{S_{L}-S_{R}}\left(u_{L}+\beta S_{M} \frac{S_{R}-S_{M}}{S_{M}-S_{L}}+\left(S_{M}-u_{L}\right)\right) .
$$

We now introduce $\beta=1$,

$$
\begin{aligned}
& (\rho u)_{L[\mathrm{RSIR}]}^{*}=\frac{\rho_{L}\left(u_{L}-S_{L}\right)}{S_{L}-S_{R}} S_{M}\left(-\frac{S_{R}-S_{M}}{S_{M}-S_{L}}-1\right), \\
& (\rho u)_{L[\mathrm{RSIR}]}^{*}=\frac{\rho_{L}\left(u_{L}-S_{L}\right)}{S_{L}-S_{R}} S_{M}\left(\frac{S_{L}-S_{R}}{S_{M}-S_{L}}\right) .
\end{aligned}
$$

Finally, the HLLC formulation is recovered,

$$
(\rho u)_{L[\mathrm{RSIR}]}^{*}=\frac{\rho_{L}\left(u_{L}-S_{L}\right)}{S_{M}-S_{L}} S_{M}=(\rho u)_{L[\mathrm{HLLC}]}^{*}
$$

Energy solution

The RSIR energy solution reads,

$$
(\rho E)_{L[\mathrm{RSIR}]}^{*}=(\rho E)_{L[\mathrm{HLL}]}^{*}-\omega_{R} \Psi^{\mathrm{energy}}
$$

with

$$
(\rho E)_{L[\mathrm{HLL}]}^{*}=\frac{[(\rho E+p) u]_{R}-[(\rho E+p) u]_{L}+S_{L}(\rho E)_{L}-S_{R}(\rho E)_{R}}{S_{L}-S_{R}}
$$

and

$$
\Psi^{\mathrm{energy}}=\beta\left(\frac{(\rho E)_{R}\left(u_{R}-S_{R}\right)+p_{R} u_{R}-p_{R}^{*} S_{M}}{S_{M}-S_{R}}-\frac{(\rho E)_{L}\left(u_{L}-S_{L}\right)+p_{L} u_{L}-p_{L}^{*} S_{M}}{S_{M}-S_{L}}\right)
$$

and

$$
\omega_{R}=\frac{S_{R}-S_{M}}{S_{R}-S_{L}}
$$


The combination of these relations leads to,

$$
\begin{aligned}
(\rho E)_{L[\mathrm{RSIR}]}^{*} & =[(\rho E+p) u]_{R} \overbrace{\left(\frac{1}{S_{L}-S_{R}}-\beta \frac{S_{R}-S_{M}}{S_{R}-S_{L}} \frac{1}{S_{M}-S_{R}}\right)}^{=0 \text { if } \beta=1} \\
& -[(\rho E+p) u]_{L}\left(\frac{1}{S_{L}-S_{R}}-\beta \frac{S_{R}-S_{M}}{S_{R}-S_{L}} \frac{1}{S_{M}-S_{L}}\right) \\
& +(\rho E)_{L}\left(\frac{S_{L}}{S_{L}-S_{R}}-\beta \frac{S_{R}-S_{M}}{S_{R}-S_{L}} \frac{S_{L}}{S_{M}-S_{L}}\right) \\
& -(\rho E)_{R} \underbrace{\left(\frac{S_{R}}{S_{L}-S_{R}}-\beta \frac{S_{R}-S_{M}}{S_{R}-S_{L}} \frac{S_{R}}{S_{M}-S_{L}}\right)}_{=0 \text { if } \beta=1} \\
& -\beta \frac{\left.S_{R}-S_{R}^{*} S_{M}\right)}{S_{R}-S_{L}}\left(\frac{p_{L}^{*} S_{M}}{S_{M}-S_{L}}-\frac{p_{R}-S_{R}}{S_{M}}\right) .
\end{aligned}
$$

HLL solutions appears recovered when $\beta=0$. However for $\beta=1$, the solution becomes,

$$
\begin{aligned}
(\rho E)_{L[\mathrm{RSIR}]}^{*}= & -[(\rho E+p) u]_{L} \frac{1}{S_{L}-S_{R}}\left(1+\frac{S_{R}-S_{M}}{S_{M}-S_{L}}\right) \\
& +(\rho E)_{L} \frac{S_{L}}{S_{L}-S_{R}}\left(1+\frac{S_{R}-S_{M}}{S_{M}-S_{L}}\right) \\
& -\frac{S_{R}-S_{M}}{S_{R}-S_{L}}\left(\frac{p_{L}^{*} S_{M}}{S_{M}-S_{L}}-\frac{p_{R}^{*} S_{M}}{S_{M}-S_{R}}\right) .
\end{aligned}
$$

Thus,

$$
(\rho E)_{L[\mathrm{RSIR}]}^{*}=\frac{[(\rho E+p) u]_{L}-S_{L}(\rho E)_{L}}{S_{M}-S_{L}}-\frac{S_{M}\left(S_{R}-S_{M}\right)}{S_{R}-S_{L}}\left(\frac{p_{L}^{*}}{S_{M}-S_{L}}-\frac{p_{R}^{*}}{S_{M}-S_{R}}\right) .
$$

As the Euler equations are considered in this paper, equality of the star pressures is now used, $p_{L}^{*}=p_{R}^{*}=p^{*}$. Relation (5.28) results in,

$$
(\rho E)_{L[\mathrm{RSIR}]}^{*}=\frac{[(\rho E+p) u]_{L}-S_{L}(\rho E)_{L}-p^{*} S_{M}}{S_{M}-S_{L}}=(\rho E)_{L[\mathrm{HLLC}]}^{*},
$$

which is once more the HLLC solution.

Similar manipulations regarding the right solution state vector provide the same conclusion,

$$
\mathbf{U}_{R[\mathrm{RSIR}]}^{*}=\mathbf{U}_{R[\mathrm{HLLC}]}^{*} \cdot
$$

It consequently appears that the reconstruction method based on,

$$
\left\{\begin{aligned}
\mathbf{U}_{L[\mathrm{RSIR}]}^{*} & =\mathbf{U}_{[\mathrm{HLL}]}^{*}-\omega_{R} \Psi \\
\mathbf{U}_{R[\mathrm{RSIR}]}^{*} & =\mathbf{U}_{[\mathrm{HLL}]}^{*}+\omega_{L} \Psi
\end{aligned}\right.
$$


is equivalent to the HLLC solver when the jump vector $\Psi$ is determined through RankineHugoniot relations. The following computed results confirm this observation. Consequently, it seems that RSIR recovers the HLLC solver (or the HLL one as another limit case) and can be degraded at different levels when complex systems of equations are considered, for example when Rankine-Hugoniot relations are not trivial, or contact wave conditions are intricate (this is true for example with two-phase flow equations). In the content of the Euler equations, the RSIR solver based on approximate thermodynamics (Section 3) provide results in close agreement with the HLLC solver as shown in the next section.

\section{Numerical results}

Comparison with the HLLC solver and exact solution is now addressed on various test problems. Let us recall that $\beta=1$ in all computations. The following test problems are given in Toro (2009) [15] (page 334). In addition, the blast wave test problem of Colella and Woodward (1984) [18] is also addressed (see also [15], page 612).

In these computations all variables are dimensionless as done in [15], [19]. Note that these test problems are quite severe except tests 6 and 7 that involve a stationary discontinuity and transport of a discontinuity. Those two last tests are nonetheless mandatory to assess methods' accuracy.

All results are provided by the Godunov (1959) [20] first-order scheme,

$$
\mathbf{U}_{i}^{n+1}=\mathbf{U}_{i}^{n}-\frac{\Delta t}{\Delta x}\left(\mathbf{F}_{i+\frac{1}{2}}^{*}-\mathbf{F}_{i-\frac{1}{2}}^{*}\right)
$$

where $n+1$ and $n$ denote two consecutive time steps and superscript ${ }^{*}$ denotes the Riemann problem solution provided by the RSIR and HLLC solvers. Indexes $i$ and $i \pm \frac{1}{2}$ denote respectively the center of the current numerical cell and its corresponding boundaries.

Obviously, higher-order extensions can be considered but add complexity to examine Riemann solvers' accuracy. Note that the Godunov scheme is stable under the conventional $C F L$ condition. For proper assessment, all results are computed using $C F L=0.9$ and a coarse mesh made of 100 regular cells. The initial conditions are provided in Table 1 . 


\begin{tabular}{cccccccc}
\hline \hline Test & $x_{0}$ & $\rho_{L}$ & $u_{L}$ & $p_{L}$ & $\rho_{R}$ & $u_{R}$ & $p_{R}$ \\
\hline 1 & 0.3 & 1.0 & 0.75 & 1.0 & 0.125 & 0.0 & 0.1 \\
2 & 0.5 & 1.0 & -2.0 & 0.4 & 1.0 & 2.0 & 0.4 \\
3 & 0.5 & 1.0 & 0.0 & 1000.0 & 1.0 & 0.0 & 0.01 \\
4 & 0.4 & 5.99924 & 19.5975 & 460.894 & 5.99242 & -6.19633 & 46.0950 \\
5 & 0.8 & 1.0 & -19.59745 & 1000.0 & 1.0 & -19.59745 & 0.01 \\
6 & 0.5 & 1.4 & 0.0 & 1.0 & 1.0 & 0.0 & 1.0 \\
7 & 0.5 & 1.4 & 0.1 & 1.0 & 1.0 & 0.1 & 1.0 \\
\hline \hline
\end{tabular}

Table 1: Initial conditions of the 7 test problems of Toro (2009) [15] page 334. The computational domain is the interval $[0,1]$ for all tests. An initial discontinuity is located at $x_{0}$. All initial conditions are dimensionless. The initial conditions of the Colella and Woodward (1984) [18] test problem are given in Fig. 9 (see also [15], page $612)$.

In the following figures, the RSIR solver based on approximate thermodynamics (Section 3) is compared to the exact and HLLC solutions. In addition, the RSIR solution based on RankineHugoniot relations is plotted as well and is referred as "RSIR RH". The solution of the original RSIR solver introduced in Carmouze et al. (2019) [8] is also plotted and is referred as "RSIR orig.". Note that the exact solution is computed with an exact solver and then plotted with 1000 points. 

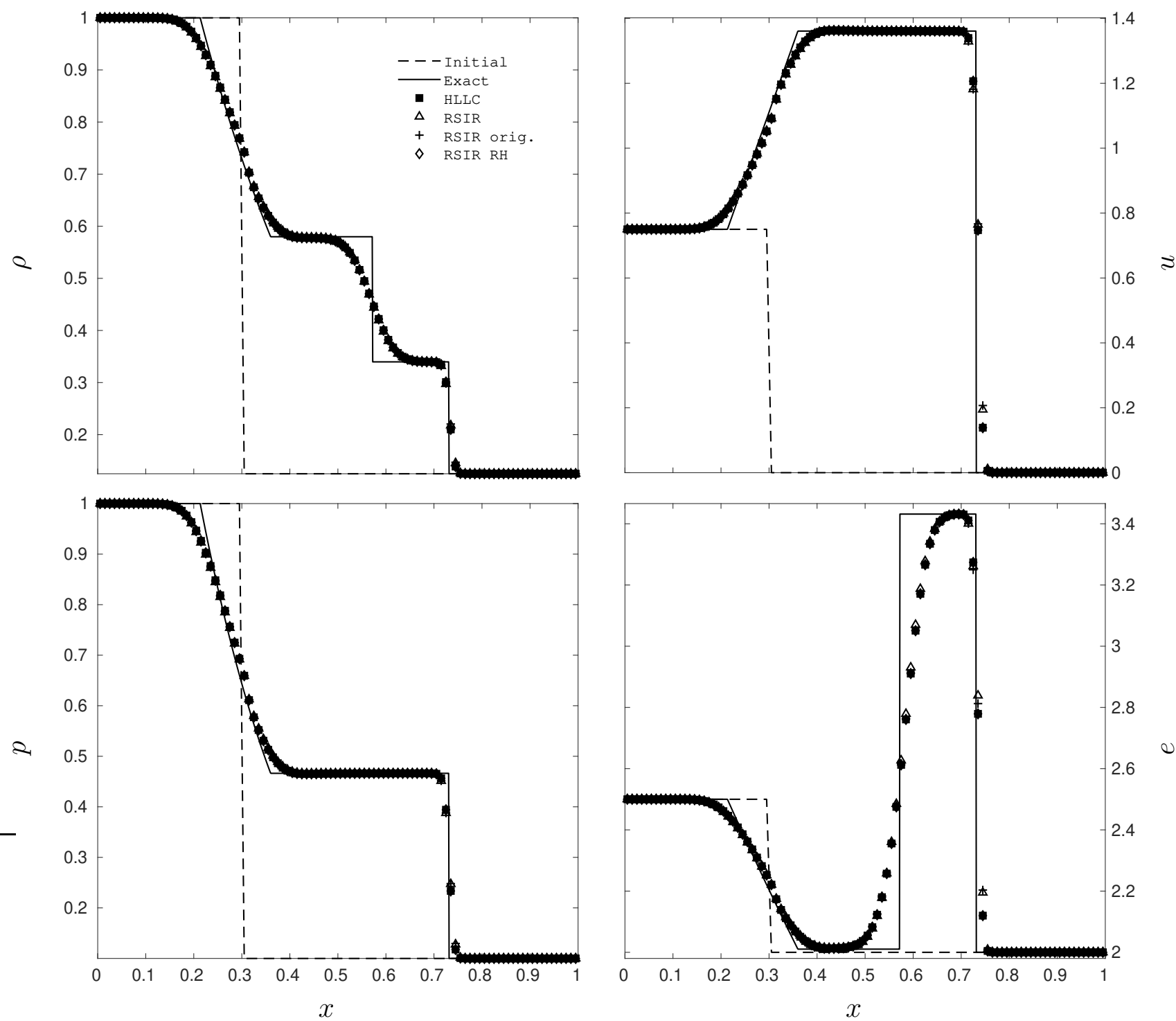

Figure 2: Test 1 of [15] page 334 (shock tube test). Comparison of the RSIR, HLLC and exact solutions. The computational domain involves 100 cells and the Godunov first-order scheme is used with $C F L=0.9$. Results are shown at time $t=0.2$. The initial discontinuity is located at $x_{0}=0.3$. Non-reflective boundary conditions are considered. All methods show comparable accuracy. 

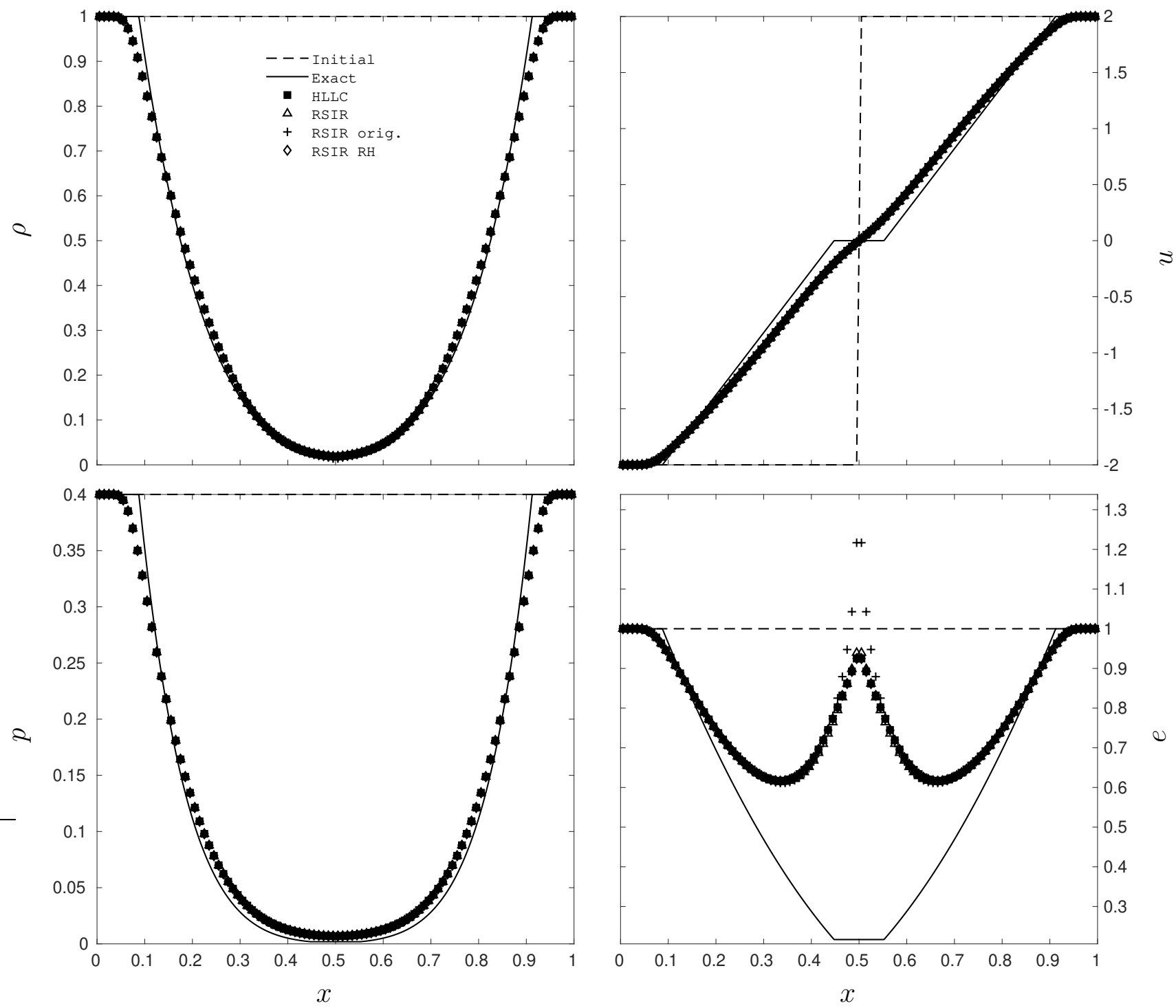

Figure 3: Test 2 of 15] page 334 (double expansion test). Comparison of the RSIR, HLLC and exact solutions. The computational domain involves 100 cells and the Godunov first-order scheme is used with $C F L=0.9$. Results are shown at time $t=0.15$. The initial discontinuity is located at $x_{0}=0.5$. Non-reflective boundary conditions are considered. All methods produce unphysical overheating at the center of the domain regarding the internal energy. The other flow variables are computed correctly. Issues related to overheating do not seem related to the Riemann solver's accuracy but more to the computation of the kinetic energy (Cocchi et al. (1998) 21]). Improvement of the present version of the RSIR solver is clearly visible in the internal energy plot, showing comparison to the original version introduced in Carmouze et al. (2019) [8]. 



Figure 4: Test 3 of 15] page 334 (strong shock tube test). Comparison of the RSIR, HLLC and exact solutions. The computational domain involves 100 cells and the Godunov first-order scheme is used with $C F L=0.9$. Results are shown at time $t=0.012$. The initial discontinuity is located at $x_{0}=0.5$. Non-reflective boundary conditions are considered. All methods show comparable accuracy. 

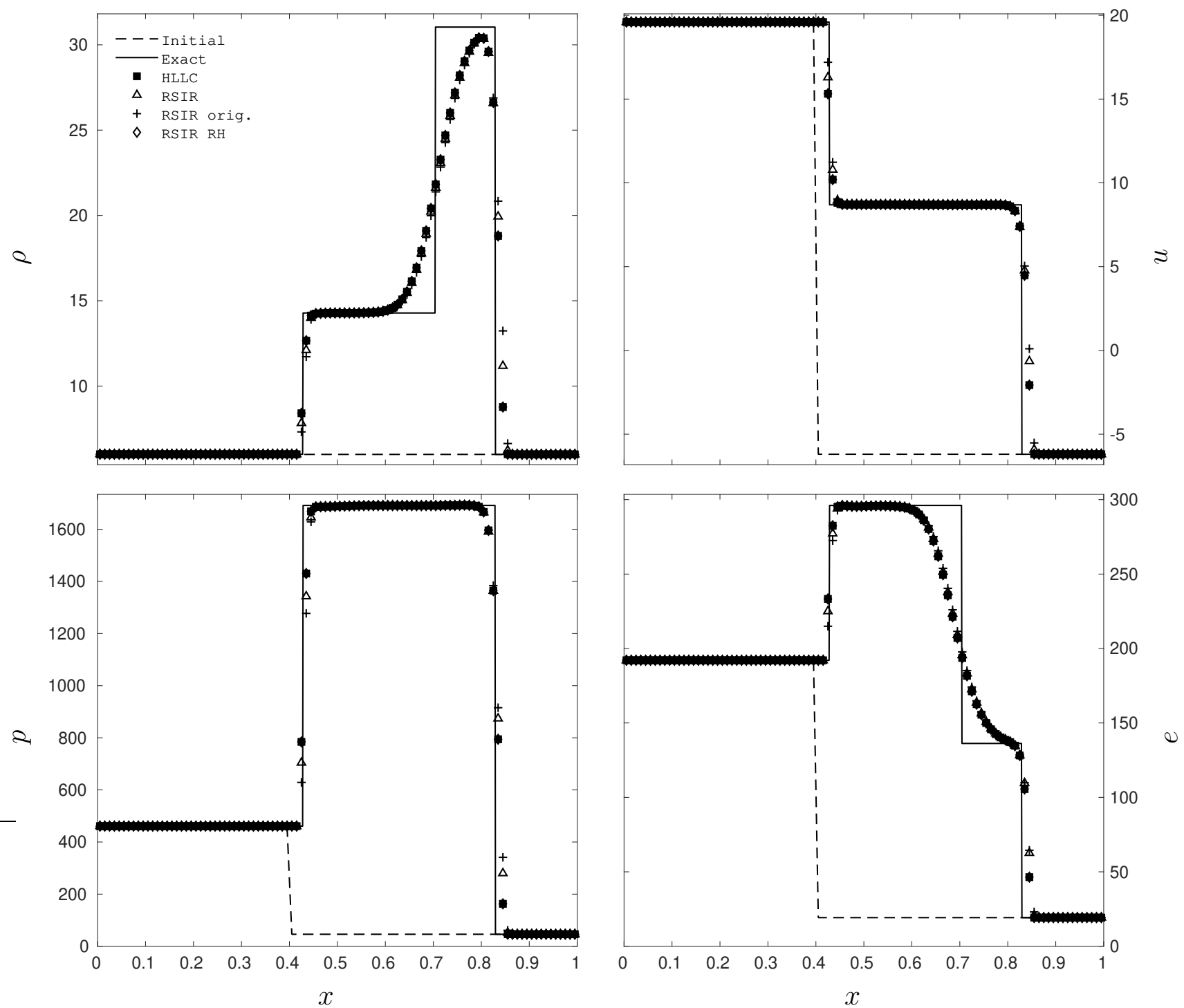

Figure 5: Test 4 of 15] page 334 (double shock test). Comparison of the RSIR, HLLC and exact solutions. The computational domain involves 100 cells and the Godunov first-order scheme is used with $C F L=0.9$. Results are shown at time $t=0.035$. The initial discontinuity is located at $x_{0}=0.4$. Non-reflective boundary conditions are considered. All methods show comparable accuracy. 

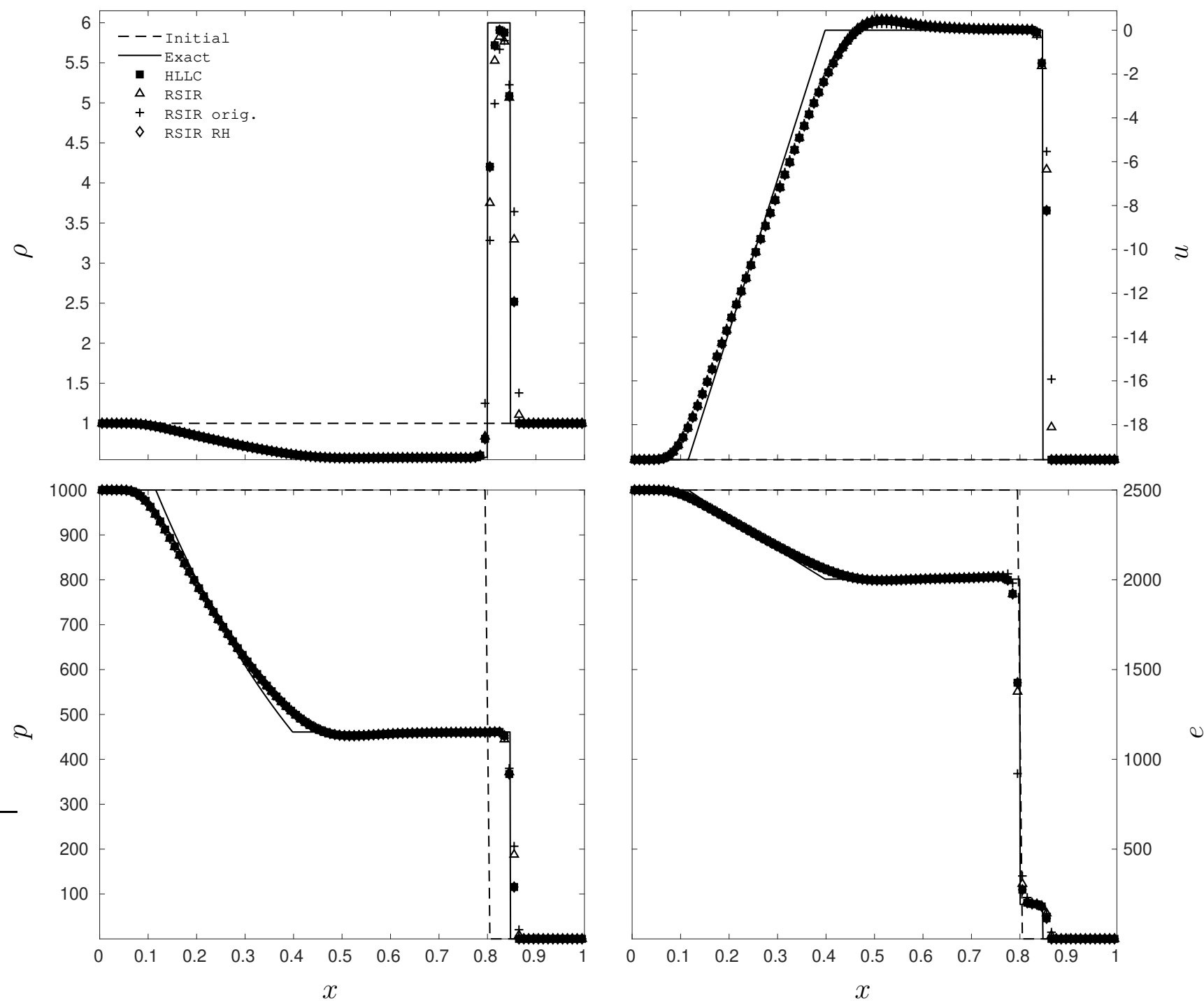

Figure 6: Test 5 of [15] page 334 (strong shock tube test of Test 3 with non-zero initial velocity). Comparison of the RSIR, HLLC and exact solutions. The computational domain involves 100 cells and the Godunov first-order scheme is used with $C F L=0.9$. Results are shown at time $t=0.012$. The initial discontinuity is located at $x_{0}=0.8$. Non-reflective boundary conditions are considered. All methods show comparable accuracy. 

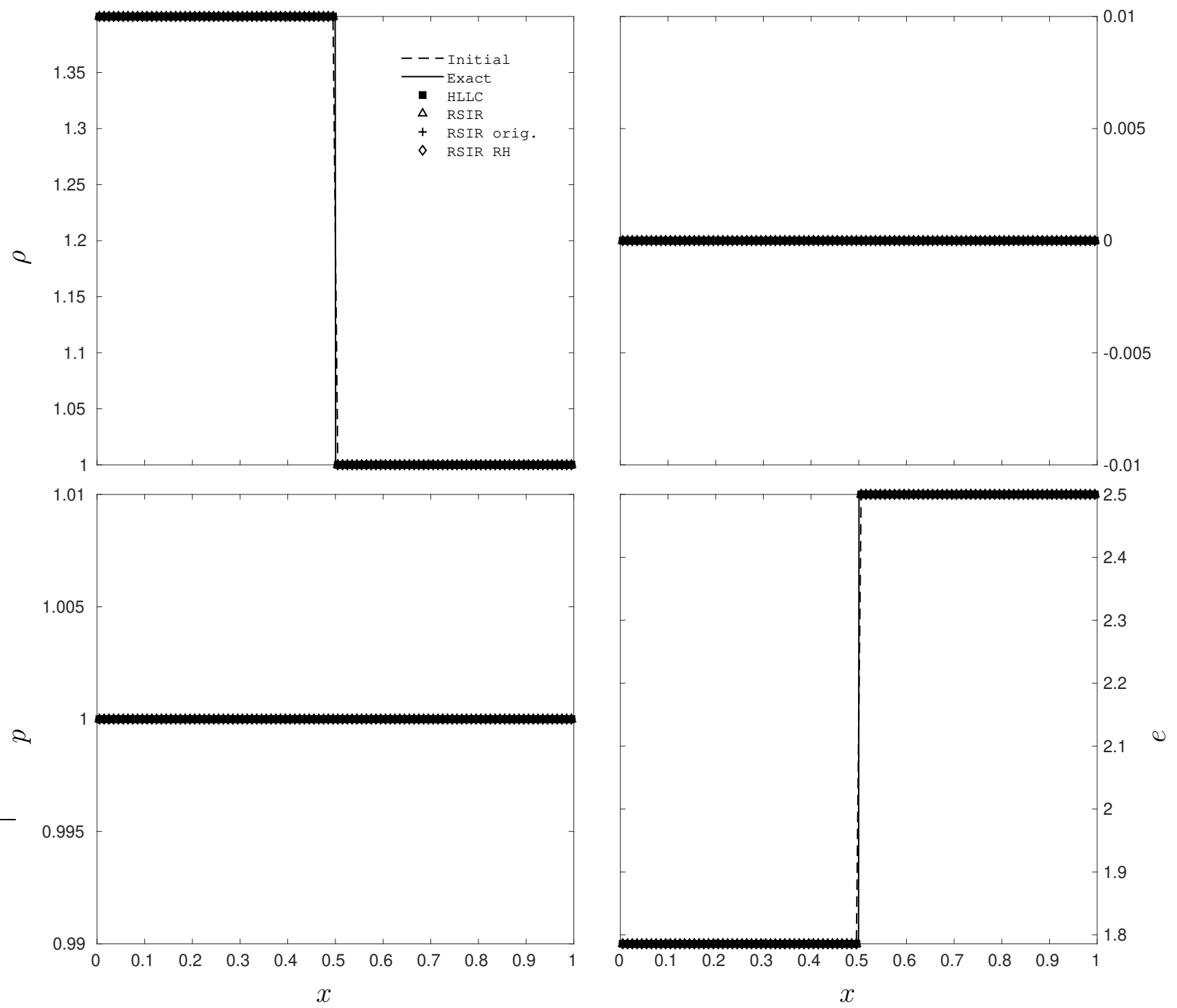

Figure 7: Test 6 of [15] page 334 (stationary contact discontinuity). Comparison of the RSIR, HLLC and exact solutions. The computational domain involves 100 cells and the Godunov first-order scheme is used with $C F L=0.9$. Results are shown at time $t=2$. The initial discontinuity is located at $x_{0}=0.5$. Non-reflective boundary conditions are considered. All methods maintain stationary contact discontinuity. 

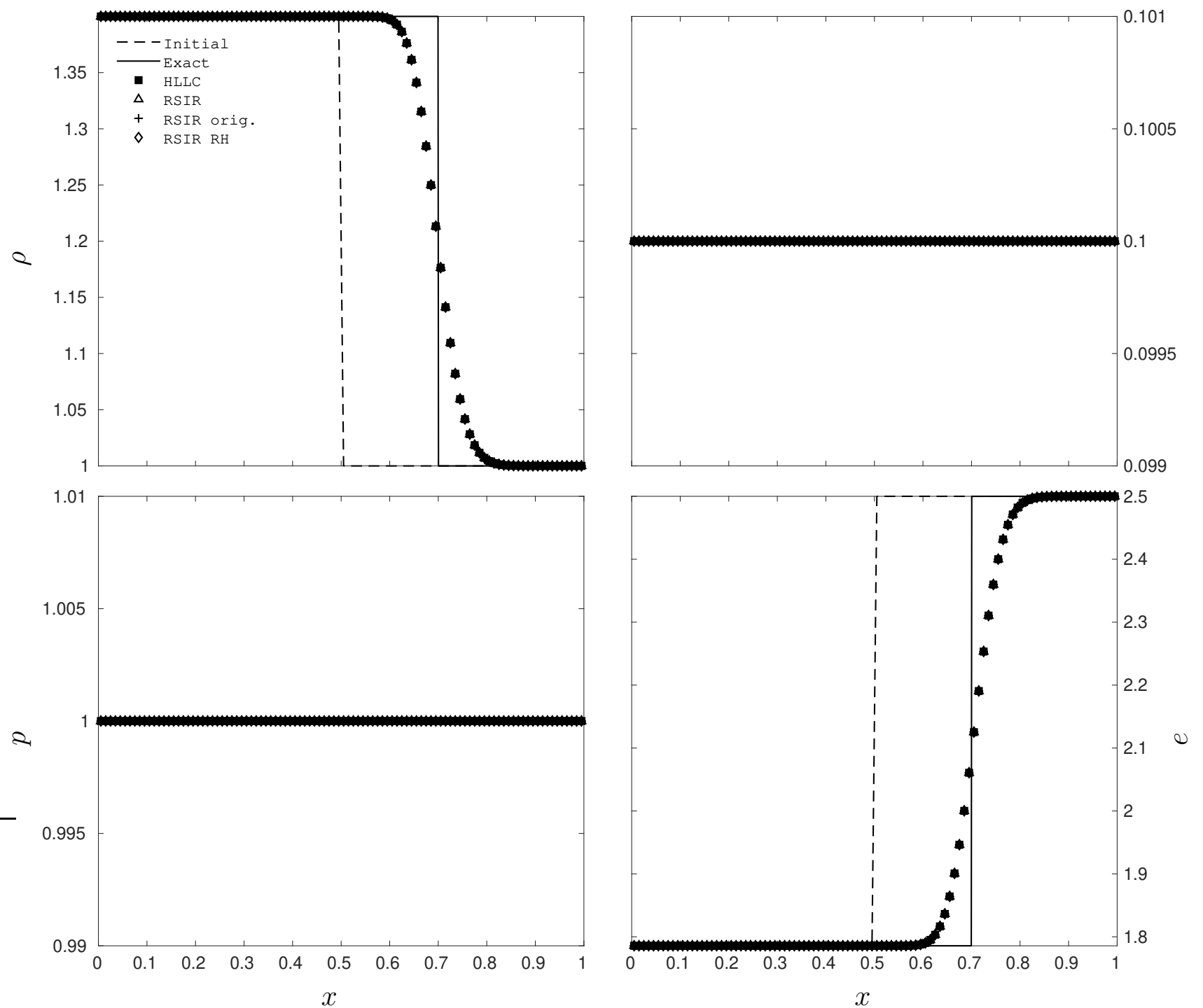

Figure 8: Test 7 of 15] page 334 (moving contact discontinuity). Comparison of the RSIR, HLLC and exact solutions. The computational domain involves 100 cells and the Godunov first-order scheme is used with $C F L=$ 0.9 . Results are shown at time $t=2$. The initial discontinuity is located at $x_{0}=0.5$. Non-reflective boundary conditions are considered. All methods show comparable accuracy. 



Figure 9: Woodward and Colella (1984) [18] blast wave test (see also Toro (2009) [15] page 612). Comparison of the RSIR and HLLC solutions. The computational domain involves 100 cells and the Godunov first-order scheme is used with $C F L=0.9$. Results are shown at time $t=0.038$. All methods show comparable accuracy. This test problem has no exact solution, but computed results with the HLLC solver and 3000 cells are shown as reference solution. The computational domain is the interval $[0,1]$. The initial condition in pressure consists of three constant states separated by two discontinuities, namely $p(x, 0)=p_{L}=1000$ for $x<1 / 10, p(x, 0)=p_{M}=0.01$ for $1 / 10<x<9 / 10$ and $p(x, 0)=p_{R}=100$ for $x>9 / 10$. Particle velocity and density are constant, with $u(x, 0)=0$ and $\rho(x, 0)=1$. Reflective boundary conditions are imposed. Note that for the sake of clarity the scale is adapted.

All tests lead to the same observations. The RSIR solver provides excellent and oscillationfree results in addition to being robust and positive. Its limit based on Rankine-Hugoniot relations provides results in perfect agreement with the HLLC solver. This observation confirms the analysis of Section 5 ,

The present RSIR solver is an improvement of the original version introduced in Carmouze et 
al. (2019) [8] as clearly seen in Fig. 3] where the unphysical overheating of the internal energy is significantly reduced, providing results in close agreement with those of the HLLC solver. This improvement is due to the introduction of Gibbs identity in the form (3.18) when computing the energy jump.

Unlike the HLLC solver, the RSIR one is based on thermodynamics and internal reconstruction. The discrete entropy production is satisfied and is quantified by the average sound speed $\bar{c}^{2}$. In all previous tests, $\bar{c}^{2}=\max \left[c_{L}^{2}, c_{R}^{2}\right]$ in order to maximize the discrete entropy production for the sake of robustness. This flexibility is an interesting feature. For instance tests 3, 4, 5 and 8 fail if $\bar{c}^{2}=\min \left[c_{L}^{2}, c_{R}^{2}\right]$ (Figs. 4, 5, 6] and 9) while tests 1, 2,6 and 7 provide similar results (Figs. 2, 3, 7 and 8).

Flexibility and simplicity of the RSIR solver are of particular interest for complicated flow models. In the context of the Euler equations, the RSIR solver recovers the HLL solver in some limit and the HLLC one in another limit.

\section{Conclusion}

The Riemann solver with internal reconstruction (RSIR) of Carmouze et al. (2019) [8] has been investigated, revisited and improved for the Euler equations. While the RSIR solver based on approximate thermodynamics produces accurate and robust results, similar to those provided

by the HLLC solver of Toro et al. (1994) [5], its limit using all set of Rankine-Hugoniot relations appears strictly equivalent to the HLLC solver.

RSIR-type solvers seem flexible for many applications where most of the physics is governed by two extreme waves and an intermediate one as they can be simplified at different levels when complex systems of equations are addressed. Two-phase flow models are for instance part of those applications as seen in Carmouze et al. (2019) [8] and Chiapolino and Saurel (2020) [14].

\section{Acknowledgements}

The authors are very grateful to Quentin Carmouze for numerous helpful discussions that definitely helped to improve the quality of this work.

\section{AppendixA. Summary of the RSIR solver}

The present appendix summarizes the RSIR formulas for a straightforward implementation. The two extreme wave speeds are first computed. Davis' estimates are used in the present contribution,

$$
S_{L}=\min \left(u_{L}-c_{L}, u_{R}-c_{R}\right) \quad \text { and } \quad S_{R}=\max \left(u_{L}+c_{L}, u_{R}+c_{R}\right) .
$$

The HLL solution state is then computed,

$$
\mathbf{U}_{[\mathrm{HLL}]}^{*}=\frac{\mathbf{F}_{R}-\mathbf{F}_{L}+S_{L} \mathbf{U}_{L}-S_{R} \mathbf{U}_{R}}{S_{L}-S_{R}} .
$$


The contact wave speed $S_{M}$ is now required. In the context of the Euler equations, it reads,

$$
S_{M}=u_{L}^{*}=u_{R}^{*}=\frac{p_{R}-p_{L}+(\rho u)_{L}\left(S_{L}-u_{L}\right)-(\rho u)_{R}\left(S_{R}-u_{R}\right)}{\rho_{L}\left(S_{L}-u_{L}\right)-\rho_{R}\left(S_{R}-u_{R}\right)}=\frac{U_{[\mathrm{HLL}]}^{*, \text { momentum }}}{U_{[\mathrm{HLL}]}^{*, \text { mass }}} .
$$

410

The two "weights" $\omega_{R}$ and $\omega_{R}$ are then computed,

$$
\omega_{R}=\frac{S_{R}-S_{M}}{S_{R}-S_{L}} \quad \text { and } \quad \omega_{L}=\frac{S_{M}-S_{L}}{S_{R}-S_{L}} .
$$

The solution densities are now determined with the help of the mass jump and the consistency relation,

$$
\left\{\begin{array}{l}
\rho_{L}^{*}=\rho_{[\mathrm{HLL}]}^{*}-\omega_{R} \beta\left(\rho_{R}-\rho_{L}+\frac{p_{L}-p_{R}}{\bar{c}^{2}}\right), \\
\rho_{R}^{*}=\rho_{[\mathrm{HLL}]}^{*}+\omega_{L} \underbrace{\beta\left(\rho_{R}-\rho_{L}+\frac{p_{L}-p_{R}}{\bar{c}^{2}}\right)}_{\Psi^{\text {mass }}},
\end{array}\right.
$$

where the average sound speed reads,

$$
\bar{c}^{2}=\max \left[c_{L}^{2}, c_{R}^{2}\right]
$$

The solution momenta are similarly obtained,

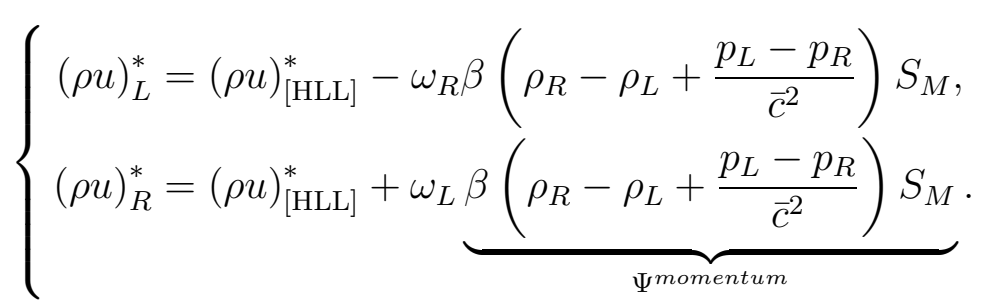

The total energies solutions are then computed,

$$
\left\{\begin{array}{c}
(\rho E)_{L}^{*}=(\rho E)_{[\mathrm{HLL}]}^{*}-\omega_{R} \beta\left[\rho_{R}^{*}\left(e_{R}^{*}+\frac{1}{2} S_{M}^{2}\right)-\rho_{L}^{*}\left(e_{L}^{*}+\frac{1}{2} S_{M}^{2}\right)\right] \\
(\rho E)_{R}^{*}=(\rho E)_{[\mathrm{HLL}]}^{*}+\omega_{L} \beta \underbrace{\left[\rho_{R}^{*}\left(e_{R}^{*}+\frac{1}{2} S_{M}^{2}\right)-\rho_{L}^{*}\left(e_{L}^{*}+\frac{1}{2} S_{M}^{2}\right)\right]}_{\Psi^{\text {energy }}},
\end{array}\right.
$$

where $\rho_{R}^{*}$ and $\rho_{L}^{*}$ are given by Eq. (A.5) and $e_{R}^{*}$ and $e_{R}^{*}$ are provided by,

$$
\left\{\begin{array}{l}
e_{L}^{*}=e_{L}-p^{*}\left(v_{L}^{*}-v_{L}\right) \\
e_{R}^{*}=e_{R}-p^{*}\left(v_{R}^{*}-v_{R}\right)
\end{array}\right.
$$


where $v_{L}^{*}=1 / \rho_{L}^{*}$ and $v_{R}^{*}=1 / \rho_{R}^{*}$ and,

$$
p^{*}=p_{L}+\bar{c}^{2}\left(\rho_{L}^{*}-\rho_{L}\right)=p_{R}+\bar{c}^{2}\left(\rho_{R}^{*}-\rho_{R}\right) .
$$

The intermediate solution states are then fully determined for the Euler equations. They are computed upon the HLL solution with the help of the consistency relation and the jump vector,

$$
\left\{\begin{aligned}
\mathbf{U}_{L[\mathrm{RSIR}]}^{*} & =\mathbf{U}_{[\mathrm{HLL}]}^{*}-\omega_{R} \boldsymbol{\Psi}, \\
\mathbf{U}_{R[\mathrm{RSIR}]}^{*} & =\mathbf{U}_{[\mathrm{HLL}]}^{*}+\omega_{L} \boldsymbol{\Psi} .
\end{aligned}\right.
$$

[6] S. Tokareva, E. Toro, HLLC-type Riemann solver for the Baer-Nunziato equations of compressible two-phase flow, Journal of Computational Physics 229 (10) (2010) 3573-3604.

\section{References}

[1] D. Balsara, A two-dimensional HLLC Riemann solver for conservation laws: Application to Euler and magnetohydrodynamic flows, Journal of Computational Physics 231 (22) (2012) $7476-7503$.

[2] S. Gavrilyuk, N. Favrie, R. Saurel, Modelling wave dynamics of compressible elastic materials, Journal of Computational Physics 227 (5) (2008) 2941-2969.

[3] R. Saurel, A. Chinnayya, Q. Carmouze, Modelling compressible dense and dilute two-phase flows, Physics of Fluids 29 (6) (2017) 063301.

[4] I. Peshkov, E. Romenski, F. Fambri, M. Dumbser, A new causal general relativistic formulation for dissipative continuum fluid and solid mechanics and its solution with high-order ADER schemes, arXiv preprint arXiv:1910.02687 (2019).

[5] E. Toro, M. Spruce, W. Spears, Restoration of the contact surface in the HLL-Riemann solver, Shock Waves 4 (1) (1994) 25-34. 
[7] D. Furfaro, R. Saurel, A simple HLLC-type Riemann solver for compressible non-equilibrium two-phase flows, Computers \& Fluids 111 (2015) 159-178.

[8] Q. Carmouze, R. Saurel, A. Chiapolino, E. Lapébie, Riemann solver with internal reconstruction (RSIR) for compressible single-phase and non-equilibrium two-phase flows, Journal of Computational Physics (2019) 109176.

[9] V. Rusanov, The calculation of the interaction of non-stationary shock waves and obstacles, USSR Computational Mathematics and Mathematical Physics 1 (2) (1962) 304-320.

[10] A. Harten, P. Lax, B. van Leer, On Upstream Differencing and Godunov-Type Schemes for Hyperbolic Conservation Laws, SIAM Review 25 (1) (1983) 35-61.

[11] B. Einfeldt, C. Munz, P. Roe, B. Sjögreen, On Godunov-Type Methods near Low Densities, Journal of Computational Physics 92 (1991) 273-295.

[12] T. Linde, A practical, general-purpose, two-state HLL Riemann solver for hyperbolic conservation laws, International Journal for Numerical Methods in Fluids 40 (3-4) (2002) 391-402.

[13] M. Dumbser, D. Balsara, A new efficient formulation of the HLLEM Riemann solver for general conservative and non-conservative hyperbolic systems, Journal of Computational Physics 304 (2016) 275-319.

[14] A. Chiapolino, R. Saurel, Numerical investigations of two-phase finger-like instabilities, Computers \& Fluids, submitted 2020.

[15] E. Toro, Riemann solvers and numerical methods for fluid dynamics: A practical introduction. Third Edition, 2009.

[16] S. Davis, Simplified second-order Godunov-type methods, SIAM Journal on Scientific and Statistical Computing 9 (3) (1988) 445-473.

[17] F. Fraysse, R. Saurel, Automatic Differentiation using Operator Overloading (ADOO) for implicit resolution of hyperbolic single phase and two-phase flow models, Journal of Computational Physics 399 (2019) 108942.

[18] P. Woodward, P. Colella, The numerical simulation of two-dimensional fluid flow with strong shocks, Journal of Computational Physics 54 (1) (1984) 115-173.

[19] E. Toro, The HLLC Riemann solver: A Review, Shock Waves 29 (8) (2019) 1-18.

[20] S. Godunov, A finite difference scheme for numerical computation of the discontinuous wave solutions of equations of fluid dynamics, Math. Sb. 47 (1959) 271-306.

[21] J. Cocchi, R. Saurel, J. Loraud, Some remarks about the resolution of high velocity flows near low densities, Shock Waves 8 (2) (1998) 119-125. 